\title{
How Discrete Patterns \\ Emerge from Algorithmic Fine-Tuning A Visual Plea for Kroneckerian Finitism
}

\author{
Ivahn Smadja (ivahn.smadja@univ-paris-diderot.fr) \\ Université Paris Diderot - Paris 7 - CNRS - UMR 7219
}

\begin{abstract}
This paper sets out to adduce visual evidence for Kroneckerian finitism by making perspicuous some of the insights that buttress Kronecker's conception of arithmetization as a process aiming at disclosing the arithmetical essence enshrined in analytical formulas, by spotting discrete patterns through algorithmic fine-tuning. In the light of a fairly tractable case study, it is argued that Kronecker's main tenet in philosophy of mathematics is not so much an ontological as a methodological one, inasmuch as highly demanding requirements regarding mathematical understanding prevail over mere preemptive reductionism to whole numbers.
\end{abstract}

Keywords: philosophy of mathematics, finitism, arithmetization, Kronecker.

\section{Introduction}

Kronecker's well-known resistance to completed infinities in mathematics is often said to be blatantly at odds with most of his own work in such heavily analytic fields as complex multiplication of elliptic curves, the so-called 'Jugendtraum', or the distribution of numbers modulo 1, lately known as Kronecker-Weyl theorem ${ }^{1}$, both of these major achievements appearing prima facie as inescapably relying on the use of the principle of continuity. Though usually portrayed as a forerunner of constructivist or intuitionistic mathematics, Kronecker's youthful mathematical fruitfulness would have been secured by ignoring the philosophical dogmas of his maturity ${ }^{2}$. Still, as stressed in his late Berlin lectures, Kronecker highly praised Dirichlet not only for his introducing analytic techniques in number theory but also for his radically challenging of Gauss's restrictive definition of arithmetic.

The delimitation required by Gauss's definition of arithmetic with respect to analysis removed from the scope of number theory the continuous magnitudes and the application of methods essentially founded on them. Such a limitation seemed to be required though at a time when one still conceived such quantities geometrically; but it has become obsolete since one has recently endeavoured to define many magnitudes stemming from mechanics or geometry regardless of this origin, by which means their purely arithmetical essence comes to the fore. If one defines for instance the transcendent $\pi$ taken from geometry by means of Leibniz's series

$$
\frac{\pi}{4}=1-\frac{1}{3}+\frac{1}{5}-\frac{1}{7}+\ldots=\sum_{n=0}^{\infty} \frac{(-1)^{n}}{2 n+1}
$$

one obtains out of this one of the most beautiful arithmetical properties of the odd numbers, namely precisely the property of determining this geometrical irrational number; and in this 
sense, the famous sentence says : numero impari deus gaudet. The terms of this series are indeed arithmetically well discriminated : they have a positive or negative sign depending on whether the denominators divided by 4 provide the remainder 1 or the remainder 3 . Therefore we have here a definition of the transcendent $\pi$ of a completely arithmetical character. ${ }^{3}$

In the following, I set out to adduce visual evidence for Kroneckerian finitism by making perspicuous some of the insights that buttress Kronecker's conception of arithmetization as a process aiming at disclosing the "arithmetical essence" which is enshrined in analytic formulas, by spotting discrete patterns through algorithmic fine-tuning. Still in this case, the visualizing should not be thought of as marring the purity of higher arithmetic any more than the geometric representation of Gaussian integers by lattice points in the plane mars the purity of higher reciprocity laws. Whereas Gauss considered such an intuitive way to get hold of the algebraic integers as providing "not so much their essence (Wesen) itself, which must be grasped in a higher and more general way, as it is for us humans the purest or perhaps a uniquely and completely pure example of their application" 4 , Jacobi's elliptic function theoretic model of Poncelet's closure theorem can be consistently presented, as will be seen below, as bringing to the fore, though clothed in a geometric garment, some important features pertaining to Kronecker's arithmetical treatment of elliptic functions.

Kronecker's work on complex multiplication of elliptic functions and his emphasis on singular moduli ${ }^{5}$, are indeed, as he himself claimed ${ }^{6}$, the source and the core of his views on arithmetization and the foundations of mathematics ${ }^{7}$, and, as such, they can be seen as the outcome of a grand broadening of the insights that can be easily grasped in Jacobi's model. In the light of such a geometric illustration, it will thus be argued, in the following, that Kronecker's main tenet in philosophy of mathematics is not so much an ontological as a methodological one, inasmuch as highly demanding requirements regarding mathematical understanding prevail over mere preemptive reductionism to whole numbers.

\section{How and why modelling proofs may advance knowledge}

Thurston (1994) convincingly argues that progress in mathematics is not so much a matter of proving theorems as a matter of advancing in any kind of significant way our understanding of mathematics. It is frequently held though that "(D) mathematicians start from a few basic mathematical structures and a collection of axioms 'given' about these structures, [that $](\mathrm{T})$ there are various important questions to be answered about these structures that can be stated as formal mathematical propositions, and [eventually that] $(\mathrm{P})$ the task of the mathematician is to seek a deductive pathway from the axioms to the propositions or to their denials" 8 . But such a "definition-theorem-proof (DTP) model" of mathematics should be discarded in Thurston's view because it fails to account satisfactorily for the very source of the questioning in mathematical practice. Since one may say that defining mathematics as what mathematicians do so as to advance mathematical understanding inevitably sounds circular, Thurston blocks the objection from the outset by outlining some recursive-like definition of mathematics as the smallest subject including natural numbers and plane 
and solid geometry, which is open to indefinite progress toward deepened understanding involving ever higher structures. "In other words, as mathematics advances, we incorporate it into our thinking. As our thinking becomes more sophisticated, we generate new mathematical concepts and new mathematical structures : the subject matter of mathematics changes to reflect how we think" 9 . This view suggests a completely different picture of what progress might amount to in mathematics. The way our thinking actually works is not to be thought of, according to Thurston, as proceeding "on a single track" but rather requires high level interactions between "separate, powerful facilities" ${ }^{10}$ such as entrenched mechanisms, both linguistic and practice-related, visual and kinesthesic sense, built-in ways of deductively extracting information from given data, processual thinking as a facility for decomposing sequences of actions in time, sense for analogies, etc. Breakthrough in mathematics thus depends much more on a significant reorganization of these facilities than the mere cumulative picture might lead us to suspect.

I claim that Jacobi's modelling of Poncelet's closure theorem in terms of elliptic functions may be seen as a advance in mathematics in Thurston's sense to such an extent that it provides a fairly tractable approach to Kronecker's way of thinking not only about elliptic functions which he dealt with along Jacobi's lines throughout his mathematical career, but also about mathematics in general. Poncelet's closure theorem presents itself first as a proposition about conics in projective geometry. Given two smooth conics $C$ and $D$, for instance two nested ellipses as in Fig. 1, Poncelet (1822) considers the following construction. Starting with any point $P_{1}$, one draws from $P_{1}$ the right tangent to $D$, call it $L_{1}$, which intersects $C$ at another point $P_{2}$. Then the tangent $L_{2}$ from $P_{2}$ to $D$ meets $C$ at $P_{3}$, etc. By iteration, one can thus generate a so-called 'Poncelet traverse' between $C$ and $D$, namely a sequence of tangents to the first conic which intersect on the second one.

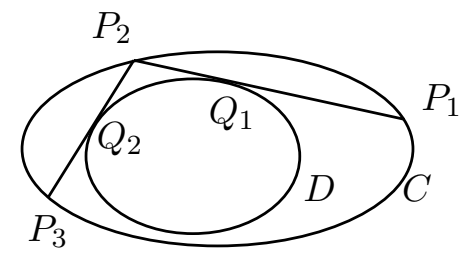

Fig. 1. Poncelet traverse

If after a finite number of steps, one happens to hit the point which started the whole process, namely if $P_{n+1}=P_{1}$, then the traverse is said to 'close', so as to determine interscribed polygons between the two conics, the so-called 'Poncelet's polygons', which may have any finite number of vertices and may wind any number of times around the ellipse (cf. Fig.2).

Poncelet's closure theorem then states that if a Poncelet traverse starting at $P_{1}$ on $C$ closes after $n$ steps, then starting from any other point on $C$, the traverse will also close after $n$ steps, or in other terms if there is an interscribed polygon between $C$ and $D$, then for every point $P$ on $C$, there is an interscribed polygon which has $P$ as one of its vertices ${ }^{11}$. Conversely, if it happens that the traverse does not close by itself, no other traverse could 
possibly close whatever the starting point. A natural question then is to ask for conditions for closure, namely what should the relations between the parameters of the two conics be so as to guarantee that interscribed polygons do close by themselves. This problem then came to be known as Poncelet's porism. While tracing out the history of Poncelet's closure theorem, Bos et al. (1987) go through a detailed scrutiny of various proofs that were given of it, from Poncelet up to twentieth-century proof in terms of algebraic geometry, and in so doing they pay a great deal of attention to the differences regarding the language in which proofs are couched and the way they are organized and conducted.
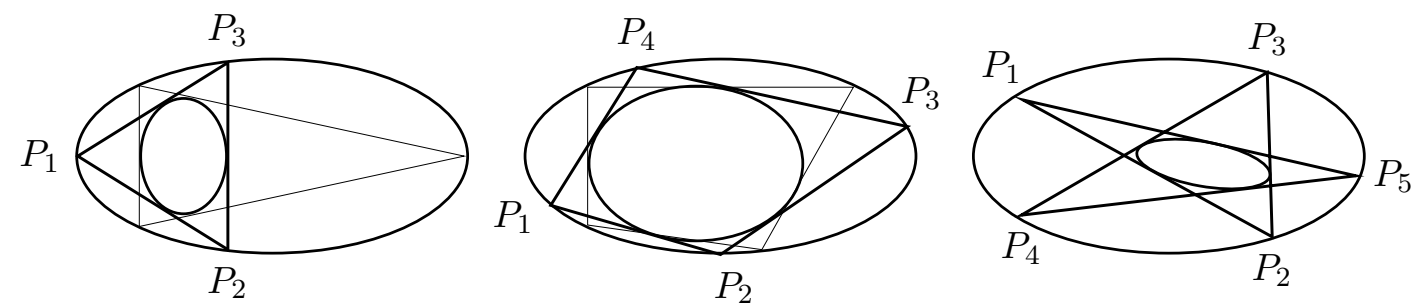

Fig. 2. Poncelet's polygons

By linking Poncelet's closure theorem with elliptic function theory, Jacobi (1828) tackled the problem from an utterly different perspective. As Bos et al. (1987) remark, Jacobi found this new approach quite incidentally while working out analytical formulas for chords and tangents in the case of a Poncelet traverse between circles. Then he hit upon certain relations that reminded him of standard formulas in elliptic function theory, so that after suitable transformations, as he himself put it, "under this form, it immediately jumps out that these equations coincide with those that hold for the multiplication of elliptic transcendents" ${ }^{12}$. Bos et al. (1987) give a full account of Jacobi's train of thought, which is rather involved and oblique since he originally aimed at couching Poncelet's geometrical notions in analytic formulas. But fortunately there is another way to get a firm grasp of how Poncelet's theorem boils down to multiplication of elliptic functions, owing to the geometric construction of elliptic functions due to Halphen. This alternative route can be seen as a shortcut not so much regarding the length of computations as regarding direct access to the main insight underlying Jacobi's approach. Halphen first devised his construction expressly as a means to facilitate the reading of Jacobi's works, inasmuch as he himself acknowledged it as bound to be eventually relegated to the background with the advent of Weierstrass's $\wp$ function ${ }^{13}$. Since Halphen's construction is derived from Jacobi's function theoretic model for Poncelet's theorem, it is indeed no miracle that it fits it so as to shed light on the property that constitutes its core, namely multiplication of elliptic functions which is to be focussed on in the following.

Consider a circle $\Gamma$ with radius $R$ and centre $O$, and a point $C$ inside the circle. From any point $M$ on the circle, draw the chord $M M^{\prime}$ through $C$, which coincides with the diameter $M_{0} M_{0}^{\prime}$ when the chord passes through the center of the circle. Halphen's idea is then to determine appropriately a certain length $C N$ along the chord $M M^{\prime}$ in such a way the area $N_{0} C N$ scanned over by the rotating variable vector $C N$ can be expressed as a function of the arc $M_{0} M$, hence of the corresponding angle $\phi=\frac{1}{2} \angle M_{0} O M$ (Cf. Fig. 3). 
Assuming that the area $N_{0} C N$ is being denoted by the variable $u$ and that the length of the rotating vector is chosen so that $C N=\sqrt{\frac{2(R+\delta)}{M M^{\prime}}}$, with $R=O M$ and $\delta=C O$, then geometric reasoning and a good deal of computation which Halphen leaves up to the reader ${ }^{14}$, bring us to the intended conclusion, namely that $u=\int_{0}^{\phi} \frac{d \phi}{\sqrt{1+k^{2} \sin ^{2} \phi}}$, which is an elliptic integral of the first kind whose modulus $k=\frac{2 \sqrt{R \delta}}{R+\delta}$ encapsulates all the relevant metrical information regarding the eccentricity of point $C$ within the circle $\Gamma$. Reversely, Halphen's construction can be used to assign geometrical content to any elliptic function with real modulus $k$, inasmuch as one just has to adjust the configuration by properly choosing the radius and the eccentricity so as to match the elliptic function ${ }^{15}$. More precisely as the point $M$ may wind indefinitely around the circle one way or another, the angle $\phi$ and the area $u$ are both supposed to vary continuously one with another so as to take all possible values from $-\infty$ to $+\infty$. Hence the area swept by the rotation of the vector $C N$ may coil up so as to superpose as many layers as one wishes. Looking now at the angle $\phi$ as a function of $u$, the periodicity of the elliptic function is made perspicuous inasmuch as the total area of the convex figure described by $C N$ when it makes a complete rotation represents a period of the function which may be denoted $2 K$.

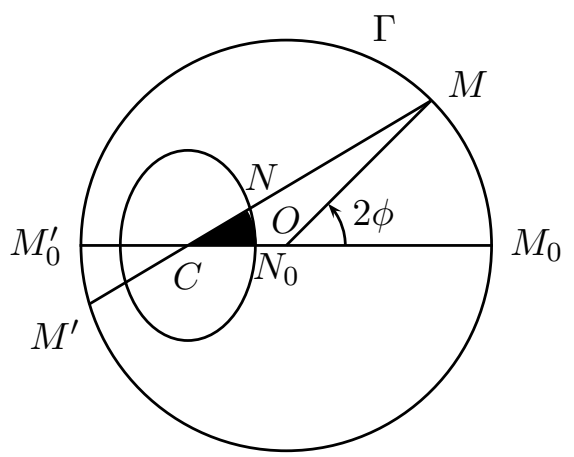

Fig. 3.

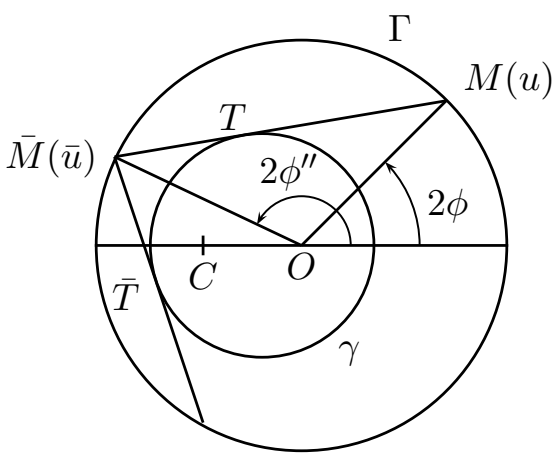

Fig. 4.

At this stage, another circle $\gamma$ lying inside circle $\Gamma$ (cf. Fig.4) has to be taken into consideration. Let it be chosen so that its center $O^{\prime}$ lies on the axis $O C$. Since there is a functional relation between the area $u$ and the angle $\phi$, any point $M$ on the circle $\Gamma$ may be assigned a given value of the argument $u$. From any point $M(u)$ on the circle $\Gamma$, one can draw a tangent at $T$ to the inner circle which can be prolonged so as to meet circle $\Gamma$ at $\bar{M}(\bar{u})$. To every inner circle $\gamma$, there corresponds a mapping $M(u) \rightarrow \bar{M}(\bar{u})$ of the circle $\Gamma$ onto itself. When comparing the values $u$ and $\bar{u}$ of the corresponding points, Halphen's line of proof consists in establishing that $d \bar{u}=d u$ and hence $\bar{u}=u+a$, where $a$ is a given constant corresponding to the choice of the inner circle. Poncelet's theorem can then be regained elegantly insofar as the traverse in progress corresponds to successive addition 
of the same constant which eventually results in multiplication if the Poncelet's polygon happens to close.

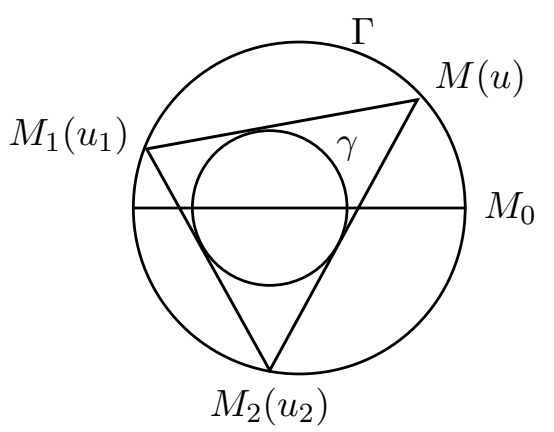

$$
\begin{aligned}
& M \quad: \quad u, \\
& M_{1}: u_{1}=u+a, \\
& M_{2}: \quad u_{2}=u+2 a,
\end{aligned}
$$

Fig. 5.. Multiplication by 3

Starting from the point $M$ with argument $u$, the polygon closes after three steps if and only if $3 a=2 K$. This construction provides one with necessary and sufficient conditions for the closure of the interscribed polygons whatever be the number of times they wind around the circle. Assuming that the traverse closes after $p$ steps and after spinning round $q$ times, both values of the argument assigned to the starting or ending point can be equated, viz. $u+2 q K=u+p a$, so that the value of the constant $a=\frac{2 q K}{p}$ can be determined. The modelling in terms of elliptic functions allows one to grasp the functional dependence between the various values of the argument, the whole pattern being preserved when the value $u$ assigned to the starting point $M$ continuously varies, which thus gives immediately Poncelet's theorem.

One of the major features of Jacobi's model consists in the correspondence according to which, along the process of constructing a traverse, geometric steps mirror exactly computational ones. This is a fundamental observation insofar as Jacobi's influence on Kronecker's ideas are concerned. As significantly noted by Bos et al. (1987), when comparing Jacobi's proof with the modern one given in terms of algebraic geometry, one might be tempted to think that similarities should count more than differences. Griffiths (1976) for instance avers that Poncelet's closure theorem is essentially equivalent to the addition law on an elliptic curve, since it presumably boils down to providing one with an elliptic curve and a point of order $n$ on it. But should one be allowed to acknowledge the elliptic curve and the group theoretic structure of an addition law as implicit in Jacobi's analytical formulas? Which content should be legitimately assigned to what proof? Caution is required since, as emphasized by Bos et al. (1987), "Jacobi himself did not explicitly introduce the structure of an elliptic curve. He proved [his] formulas constructively by direct computation. Griffiths' proof, on the contrary, is not constructive; it first recognizes $E$ as an elliptic curve and then uses existence theorems to conclude that there is an additive structure" 16 .

If computations are 'frozen hard' deep inside the abstract additive structure on an elliptic curve, powerful theorems of algebraic geometry certainly make them dispensable, since it would be pointless to compute painstakingly if one can jump to conclusions. Still, something would thereby be lost, which is precisely of paramount importance if the meaning 
of Kroneckerian finitism is to be properly assessed. So as to get hold of the importance attached to the 'singular moduli' in Kronecker's view, it will suffice to take heed of their geometric counterpart. By mentally infusing motion into the figure, one can grasp intuitively how the geometric construction is amenable to closure by apprehending how Poncelet traverse is dynamically responsive to continuous change of the inner circle. Given any two fixed circles $\Gamma$ and $\gamma$ (cf. Fig.6.), any point $M$ on the outer circle $\Gamma$ determines a Poncelet traverse $M M_{1} M_{2} M_{3}$ whose sides are tangent to the inner circle $\gamma$. But now, by continuously changing $\gamma$ either by moving its center $O$ on the diameter $A B$ of $\Gamma$ or by increasing or decreasing the length of its radius, one can shift the whole Poncelet traverse $M M_{1} M_{2} M_{3}$, like an articulated arm, so as to obtain closure by bringing the endpoint $M_{3}$ to coincide with the starting point $M$.
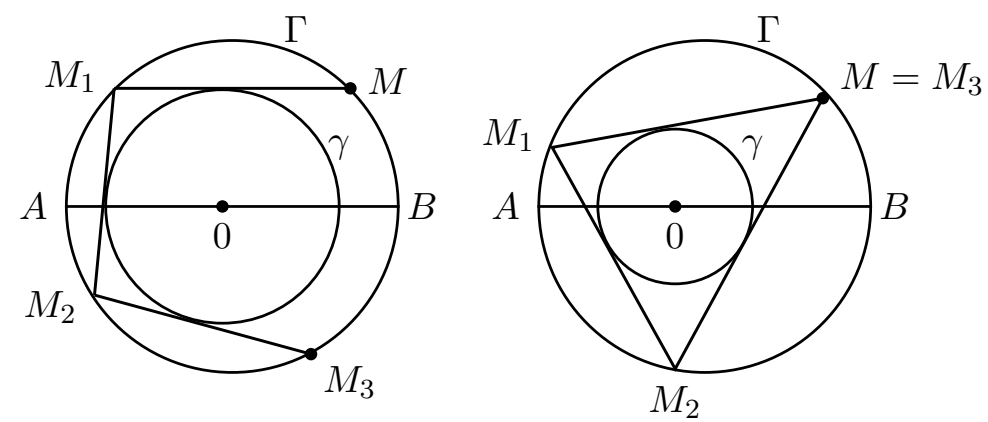

Fig. 6.. Fine-tuning the multiplication scheme

Whereas, in the vivid geometric illustration afforded by Poncelet's theorem, the inner circle may thus be freely shifted and rescaled so as to make the polygons close, on the computational side, this shifting-and-rescaling process corresponds to the adjusting of the elliptic integrals so as to make them fit for multiplication by an appropriate multiplier, which eventually results in discrete patterns emerging through algorithmic fine-tuning.

\section{Epistemic virtues}

Epistemic virtues might be conflicting in this case, insofar as purity seems to be at odds with explanatory power. Though elliptic functions shed light on Poncelet's theorem, it has been held that such insights can only be gained at the cost of utterly denaturing the theorem by divesting it of its geometric quality. Poncelet for instance resisted Jacobi's interpretation in terms of elliptic functions, on the grounds that it would appear "rather unnatural, and even more unphilosophical" 17 to draw a posteriori a proof of a simple geometrical proposition from the properties of the elliptic functions, because "the purely implicit or algorithmic character pertaining to their presentation makes the simple depend on the compound or the transcendent, contrary to the logical and rational order" ${ }^{18}$. The peculiar blending of geometrical, transcendental and algebraic ingredients that characterizes Jacobi's model certainely qualifies it as a perplexing case. As noted by Bos et al. (1987), "the fact 
that transcendental relations, namely elliptic functions, entered the proofs of a theorem which concerns purely algebraic geometrical objects, namely conics, led mathematicians to attempt purely algebraic proofs for the closure theorem, or to study the deeper reasons for the appearance of transcendental relations in this case" ${ }^{19}$. Cayley's search of an algebraic expression for the closure condition in terms of invariant theory thus started the whole process of such algebraization. At the end of his paper, Jacobi had indeed expressed the wish that the approach he had initiated be generalized from circles to arbitrary ellipses, however complicated the elliptic integral might get ${ }^{20}$. Using homegeneous coordinates, Cayley thus starts with two quadratic equations in three variables $x_{1}, x_{2}, x_{3}$, namely $U=0$ and $V=0$, which represent two given conics having four points of intersection, and by considering the pencil of conics $U+\zeta V=0$ passing through these four intersection points, he devises a method to obtain the invariant whose vanishing expresses the fact that the two conics $U=0$ and $V=0$ admit of an inscribed-and-circumbscribed polygon of a given number of sides. Though "based on complicated identities from elliptic functions" ${ }^{21}$, Cayley's method led to a fairfly simple algebraic result. Whereas in a first memoir (1853a), the properties of the pencil of conics are linked ${ }^{22}$ with an elliptic integral of the form $\int \frac{d x}{\sqrt{(x+a)(x+b)(x+c)}}$, where $D=(x+a)(x+b)(x+c)$ is the discriminant of the two base quadratic forms $U=0, V=0$, it is later shown (1853b) that the invariant can be obtained by considering the expansion of the square-root of the discriminant $D$ (denoted $\square \zeta$ ) into a series of powers of the parameter $\zeta$ :

$$
\sqrt{\square \zeta}=A+B \zeta+C \zeta^{2}+D \zeta^{3}+E \zeta^{4}+\ldots
$$

The invariants can then be written as symmetrical determinants such that (1) the order of these is equal to the number of sides of the Poncelet's polygon minus 1 or 2 depending on whether this number is even or odd, and (2) the elements of the determinants are the coefficients of the power expansion of the square-root of the discriminant. Hence for a polygon with $n$ sides, the conditions $n=3,5,7, \ldots$ yield respectively the invariants ${ }^{23}$ :

$$
|C|=0, \quad\left|\begin{array}{ll}
C & D \\
D & E
\end{array}\right|=0, \quad\left|\begin{array}{ccc}
C & D & E \\
D & E & F \\
E & F & G
\end{array}\right|=0 \quad, \ldots
$$

while the conditions $n=4,6,8, \ldots$ correspond respectively to

$$
|D|=0, \quad\left|\begin{array}{ll}
D & E \\
E & F
\end{array}\right|=0, \quad\left|\begin{array}{ccc}
D & E & F \\
E & F & G \\
F & G & H
\end{array}\right|=0 \quad, \ldots
$$

With hindsight, modern interpretations have tried to make sense of Cayley's intricate line of thought through elliptic functions by translating it into the language of Riemann surfaces $^{24}$, but the algebraic simplicity of the closure conditions was as perfect as one would wish from the outset. However, a good deal of transcendent lumber still comes into the picture along the pathway leading to the invariants. A further step is then taken with Pasch 
\& Rosanes (1869) who envisage the closure properties in a new light by recognizing as the true foundations for the whole theory, the symmetric doubly quadratic equations of the form

$$
F=A y^{2}+2 B y+C=A^{\prime} x^{2}+2 B^{\prime} x+C^{\prime}
$$

where $A, B, C$ are quadratic polynomials in $x$ whereas $A^{\prime}, B^{\prime}, C^{\prime}$ are quadratic polynomials in $y$. From these, one can indeed regain both the geometry of Poncelet traverses ${ }^{25}$ on the one hand and the elliptic functions through the Euler equation ${ }^{26}$ on the other hand, without though giving such non algebraic properties precedence over the algebraic ones.

This algebraization of the closure conditions through the progressive merging of Poncelet's theorem with elliptic functions can be interpreted along Kroneckerian lines, inasmuch as Kronecker (1891) considered the process of arithmetization as an endeavour to grasp mathematical concepts in terms of invariants thus reflecting non arithmetical properties in arithmetical one ${ }^{27}$. In any field of mathematics where equivalence relations can be devised, invariants can be defined as "functions on elements which have the same value for the whole class of equivalent systems" ${ }^{28}$. Kronecker then distinguishes three main kinds of invariants, namely "as arithmetical, algebraic and analytic, according to the method by which they are generated out of the elements of the system" ${ }^{29}$, namely depending on whether the function yielding the invariant can be embodied in a full-fledged algorithm, or is an algebraic function of the elements (and even, in best cases, a rational function of them), or eventually encapsulates limiting processes. In the case of non arithmetical fields, the process of arithmetization depends on the ability to establish through invariants an illuminating correspondence between the arithmetical and the non arithmetical properties ${ }^{30}$. As a typical instantiation of the main stream in invariant theory, algebraic geometry and elliptic function theory of the time, Cayley's algebraization of Poncelet's closure properties then provides an example of such a process. Though Cayley's invariants for Poncelet's closure property appear at first as algebraic invariants according to Kronecker's classification, they may enhance an arithmetical understanding of the geometrical fact. In the last of the series of papers devoted to the inscribed-and-circumscribed polygons, Cayley (1861) remarks indeed that when the number of sides of a polygon is a compound number, the invariant formed according to the determinant rule can be factorized into the invariants corresponding to the polygons in which the number of sides is a divisor of this compound number. Geometrical and arithmetical properties are thus brought into some sort of reflective correspondence which can be viewed both ways. Whereas a geometer would, along Poncelet's lines, claim that "a most simple geometric consideration immediately displays the reason of this algebraic fact" 31 , an arithmetical lotus-eater ${ }^{32}$ would on the contrary find in the arithmetic-algebraic properties the rational justification for the geometrical fact.

However the paradigm case Kronecker had in mind for arithmetization when devising his late philosophy of mathematics remains the joint development of elliptic function theory and number theory through quadratic forms, stemming from his early results on complex multiplication. As Hermite, who was once Kronecker's closest comrade-in-arms, assessed it, "the main work of M. Kronecker was to find in the theory of the complex transformation [namely of elliptic functions] an access more difficult but also infinitely more fruitful to 
Arithmetic. (...) M. Kronecker establishes that to every class of quadratic forms corresponds a singular modulus which allows complex multiplication (...) [he] made completely clear that the theory of quadratic forms of negative determinant was a forerunner of the theory of elliptic functions" ${ }^{33}$.

At this stage, the issue of the alleged relevance of Poncelet's closure theorem to a faithful assessment of Kronecker's thought should be raised for closer consideration. How would it be possible to trace a heuristic path from Jacobi's model to Kronecker's work? Actually, with hindsight, there are two ways to do so, insasmuch as a bifurcated development pertaining to multiplication of elliptic functions can be seen as rooted in Jacobi's model.

On the one hand, one could pursue the matter further in search of a more general statement by asking what would happen if the ongoing Poncelet's polygons never close. Since, as one might guess, such polygons would then densely fill out the circle with vertices, one recognizes a refined version of Kronecker-Weyl theorem, which, as will be seen, makes the real line dispensable, insofar as the distribution of the vertices is governed by strictly finitistic Diophantine approximation procedures.

On the other hand, Jacobi's model can be generalized in another direction by shifting from real multiplication to complex multiplication of elliptic functions, so that the discrete patterns emerging from algorithmic fine-tuning on elliptic integrals would then be focussed on in the extended setting of the complex plane, thus making the connection with lattices, quadratic forms, algebraic number fields and eventually Kronecker-Weber theorem, more at hand ${ }^{34}$. Following ideas which can be traced back to Abel, Kronecker (1857) succeeded in getting hold of the algebraic-arithmetic relations which govern complex multiplication of elliptic functions without losing sight of the computational import of the formulas ${ }^{35}$. Taking for granted what Jacobi had made clear, namely that Poncelet's theorem amounts to the multiplication of elliptic functions, one further step remains to be taken so as to get a clear enough view of Kronecker's ideas on arithmetization at its apogee, namely as regards the intimate connection between number theory and elliptic functions. Whereas Jacobi had dealt with multiplication with a real multiplier, Kronecker concentrates on complex multiplication of elliptic functions as boiling down to a singular case of the transformation of elliptic integrals for a given algebraic substitution $F(x, y)=0$, viz.

$$
F(x, y)=0 \quad \text { iff } \quad \int_{0}^{x} \frac{d x}{\sqrt{1-x^{2}} \sqrt{1-\kappa^{2} x^{2}}}=M \int_{0}^{y} \frac{d y}{\sqrt{1-y^{2}} \sqrt{1-\lambda^{2} y^{2}}}
$$

In other words, he endeavours to characterize 'singular moduli' of elliptic functions as those allowing complex multiplication, namely as those for which transformation results in both moduli $\kappa$ and $\lambda$ being the same. On the whole, transformation of elliptic functions is no more than a matter of making substitutions, hence a matter of computations, but the way in which these computations are to be organized is dictated by number theory. When performing the substitution $F(x, y)=0$ in the first integral above, one obtains the second one, which not only may be of a different nature, viz. of a different modulus $\lambda \neq \kappa$, but happens to be multiplied by a quantity brought out by the very substitution, viz. the multiplier $M$. The whole problem is to find how transformation of elliptic integrals is governed by arithmetical concerns ${ }^{36}$. Hence the central idea behind complex multiplication is to turn the 
transformation into some sort of multiplication by fine-tuning the substitutions thus making the two modules $\kappa$ and $\lambda$ coincide, or, in more vivid terms, by clinging to very same lattice in the complex plane. In order to understand how arithmetic then comes into the picture, it will suffice to give an example. If one takes for instance the two moduli $\kappa=\lambda=\sqrt{-1}$ and the multiplier $M=2$, one obtains an analytic equation, $\int_{0}^{x} \frac{d x}{\sqrt{1-x^{4}}}=2 \int_{0}^{y} \frac{d y}{\sqrt{1-y^{4}}}$, whose geometric counterpart, which launched the whole trade of elliptic functions, consists in the measuring of the arc of the Lemniscate. Though it originally took Fagnano and Euler a great deal of ingenuity to devise which substitutions were to allow for such a doubling of the Lemniscatic arc, once steadily established, these equations gave access to the whole theory of the division of the Lemniscate, which would eventually prove to be regulated by the arithmetic of the Gaussian integers just as cyclotomy is regulated by the arithmetic of rational integers. Complex multiplication of elliptic functions would then appear as the appropriate access to further extensions of arithmetic. By geometrically mirroring how multiplication, which is to be conceived in the end as arithmetical multiplication in a properly extended domain, can be obtained through a process of adjusting transformations of elliptic functions, Jacobi's model thus adumbrates how later Kronecker's finitistic stance would be inseparable from his longstanding quest for deep arithmetization of mathematics.

The remainder of the present paper will leave aside this second line of development for a later occasion, while attempting to address the first.

\section{Whirling around the circle makes the real line dispensable}

Billiards forms a thriving field of contemporary mathematics combining various methods ranging from algebraic geometry to dynamical systems ${ }^{37}$, whose attractiveness partly lies in the interplay between structural features of orbits and qualitative geometric properties of Euclidean domains, namely between the periodicity (or non-periodicity) of paths and the shape of given geometric contours. As an idealisation of a mechanical configuration, a mathematical billiard consists of a given - say convex - domain $D$ in the plane and a point moving freely along a straight line inside $D$ until it strikes the boundary $\partial D$ where it bounces in such a way that the angle of incidence equals the angle of reflection with respect to the tangent to the contour at the contact point. The moving point may be seen as a billiard ball flying inside the domain at a uniform speed which breaks down into a constant tangential and a flapping normal component which swaps sign instantaneously upon reflection, or equivalently as a light ray bouncing inside a curved mirror. A vast array of questions stemming from such configurations focus on the following issues. How much sensitive trajectories might be to the shape of the boundary. Namely, for instance, which convex domains bring out periodic paths? How do concave ones imply ergodicity by dispersing beams of light or pencils of trajectories? etc.

Poncelet's closure theorem then allows for a nice mechanical interpretation in the case of two confocal ellipses (Cf. Fig. 7). If starting from a point on the outer ellipse, the billiard ball is initially thrown in the direction of the tangent to the inner ellipse, then the whole subsequent billiard trajectory will unfold along a Poncelet traverse. However the full extent 
of Poncelet's theorem requires that the above restriction be removed owing to the obtaining of any two arbitrary nested ellipses by projective transformation from a pair of confocal ones. By replacing ellipses by circles for the sake of the argument, Jacobi's generic case (cf. Fig. 9) can then be backtracked to two concentric circles (cf. Fig. 8), so as to elicit a new reading of the whole configuration.

Since Poncelet's trajectories close up after a finite number of steps inasmuch as they can be traced back to a rigid rotation on the unit circle, the whole approach may be reversed and Jacobi's interpretation of Poncelet's theorem can be seen as the building of an invariant measure which ends up with an elliptic function. The righthand tangent to the inner ellipse (or circle) drawn from any point $z$ to the corresponding point $R z$ on the outer ellipse (or circle) can be seen as a transformation $R$ mapping the ellipse to itself. Starting from the observation that the arclength measure is $R$-invariant ${ }^{38}$ in the case of concentric circles (cf. Fig.8), King (1994) suggests that whereas in the general case, the $R$-map shrinks or stretches the subintervals of the unit circle, making thus the arclength measure squarely non invariant, an analogous $R$-invariant measure $\mu$ could be built though if only one could compensate such distorsions by complementing the arclength with an appropriate weight function $h$ suitably devised to systematically 'undo' the infinitesimal stretchings and shrinkings induced by the shift of the inner circle (cf. Fig. 9). The measure $\mu$ thus meant to replace the arclength would then be defined as $\mu(A)=\int_{A} h(z) d z$ for every subset $A$ of the unit circle. By making clear the properties that should hold of such a function $h$, the framing out of the measure in the general case would bring back the elliptic functions ${ }^{39}$.

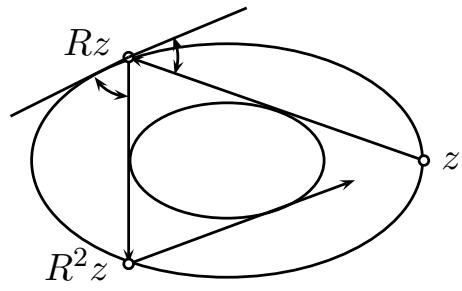

Fig. 7. Confocal ellipses

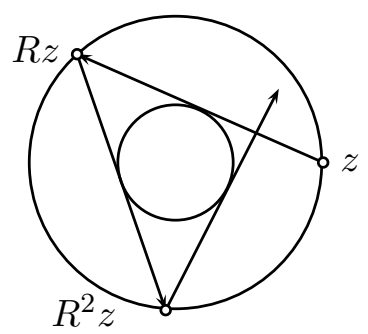

Fig. 8. Concentric circles

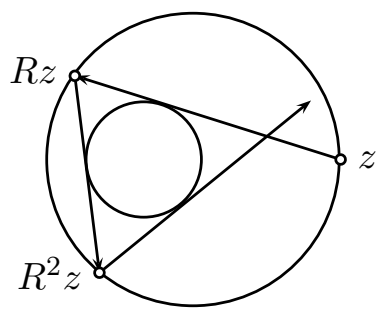

Fig. 9. Jacobi's generic case

Whereas periodicity of Poncelet's trajectories boils down to rational rotations, in the case of irrational rotations, Poncelet's ongoing polygons keep winding around the circle without ever closing up, thus filling it out with vertices. The core property underlying such a visual account is provided by Kronecker's theorem stating that under an irrational rotation $\rho_{\alpha}$, the orbit of the origin is dense in the unit circle. The proof of the theorem ${ }^{40}$ relies ultimately on a fundamental combinatorial fact, known as 'pigeonhole' principle, which avers that if $n+1$ objects are put into $n$ different boxes, there will be at least two objects in one single box. However obvious this statement may seem at first sight, Rota (1986) underscores that many of its applications may prove startling. "The reason for this is that the principle asserts that an object having a certain property exists, without giving us a means for finding such an object; however, the mere existence of such an 
object allows us to draw concrete conclusions" 41 . Inasmuch as finite collections are dealt with, the alleged unfullfilled existential statement does not mar the concreteness of the resulting propositions. Combinatorial theory should not be thought of as restricted to the mere study of finite sets though, since "infinity has a way of getting into the most finite considerations" whereas "in the ever-present interaction of finite and infinite lies the fascination of all things combinatorial" 42 . While dismissing the alleged Kroneckerian claim that "God created the integers, everything else being man-made", for no narrow finitism would ever fit combinatorial theory, Rota humorously suggests that a more accurate description might rather be "God created infinity, and man, unable to understand infinity, had to invent finite sets" 43 . However, contrary to a false legend too often credited, Kronecker himself forcefully recommended not to mark out arithmetic not only from algebra but also from analysis, provided this last science be emancipated from geometry as its original source and incentive, inasmuch as analytic number theoretic methods could enlarge the realm of arithmetic by disclosing arithmetical properties otherwise hidden. Therefore in this particular case, if mimicking Kronecker's word be allowed ${ }^{44}$, it is probably a matter of Taktgefühl to decide whether mathematical facts involving a presumably given infinite are to be accounted for combinatorially, or the other way around by dispensing at the outset with completed infinities while trying to figure out how combinatorial finitistic methods may be pushed ahead to the outmost extent.

\section{Digging out the arithmetical gist}

Looking at Poncelet's theorem as a statement about the dynamics of a given map may shed light on the significance of such deep quasi-combinatorial facts for analytic number theory. King (1994) shows indeed highly interestingly how certain number theoretic issues, such as the so-called Gelfand's question, boil down to the search for an invariant measure and eventually prove isomorphic to Poncelet's theorem. What is referred to in contemporary literature on ergodic theory of dynamical systems ${ }^{45}$ as the 'Gelfand's question' actually embraces a bunch of problems pertaining to patterns occurring in arrays of high-order digits of powers. Assuming that $\ll \alpha \gg$ denote the leftmost digit in the decimal expansion of the real number $\alpha$, so that for instance $\ll 7529 \gg=7$, one may focus on the powers of natural numbers, such as $\ll 2^{n} \gg$, $\ll 3^{n} \gg$, etc. and arrange them in a tabular as follows.

$\begin{array}{lcccccccc} & 2^{n} & 3^{n} & 4^{n} & 5^{n} & 6^{n} & 7^{n} & 8^{n} & 9^{n} \\ \text { seed : } & 1 & 1 & 1 & 1 & 1 & 1 & 1 & 1 \\ n=1 & 2 & 3 & 4 & 5 & 6 & 7 & 8 & 9 \\ n=2 & 4 & 9 & 1 & 2 & 3 & 4 & 6 & 8 \\ n=3 & 8 & 2 & 6 & 1 & 2 & 3 & 5 & 7 \\ n=4 & 1 & 8 & 2 & 6 & 1 & 2 & 4 & 6 \\ n=5 & 3 & 2 & 1 & 3 & 7 & 1 & 3 & 5 \\ \vdots & & & & & & & & \end{array}$


A whole range of cascading questions may then be asked about possibly emerging patterns, such as the following, picked out from (King, 1994, p. 610). Will a ' 9 ' ever occur in the 2-column? Is the sequence ' 23456789 ' bound to appear again beside the first row? If it is the case, does it have a frequency? And if it does, is this frequency rational or irrational? Will a row formed of exactly one repeated digit ever appear? etc. Frequencies in particular must be taken heed of, insofar as they prove to disclose unexpected features. By freely removing the bound $N$ as far as wished, the approximate value of the frequency of any given digit in any given column may be computed, say for instance the frequency of ' 8 ' in the 3-column

$$
F r_{3}(8)=\lim _{N \rightarrow \infty} \frac{1}{N}\left|\left\{n \mid 1 \leqslant n \leqslant N \& \ll 3^{n} \gg=8\right\}\right|
$$

Surprisingly enough, every digit $d$ occurs with the same frequency in every column. But joint frequencies are perhaps even more tantalizing for they provide empirical evidence for presumably deep properties. Computing joint frequencies such as the frequency of rows displaying at the same time a ' 2 ' in column 3 , a ' 1 ' in column 5 and a ' 7 ' in column 9 , viz. $\mathrm{Fr}_{3 ; 5 ; 7}(2,1,7)$, actually reveals striking features. For instance some columns prove to be independent while some do not. Why should it be the case? But still perplexing facts may be adduced with computer aided heuristic exploration by "varying the seed", as King (1994) puts it. One may indeed replace the input sequence formerly made of ' 1 ' by any other finite sequence of numbers $\left(s_{2}, s_{3}, \ldots, s_{9}\right)$, and see what happens.

$\begin{array}{ccccccc} & 2^{n} & 3^{n} & 4^{n} & 5^{n} & \ldots & 9^{n} \\ \text { seed : } & s_{2} & s_{3} & s_{4} & s_{5} & \ldots & s_{9} \\ n=1 & \ll 2 . s_{2} \gg & \ll 3 . s_{3} \gg & \ll 4 . s_{4} \gg & \ll 5 . s_{5} \gg & \ldots & \ll 9 . s_{9} \gg \\ n=2 & \ll 2^{2} . s_{2} \gg & \ll 3^{3} . s_{3} \gg & \ll 4^{2} . s_{4} \gg & \ll 5^{2} . s_{5} \gg & \ldots & \ll 9^{2} . s_{9} \gg\end{array}$

Whereas at first sight, the seed does not seem to affect one-column frequencies, since they appear to be the same for every column whatever be the input, King (1994) reports the amazing fact that not only does this independence break down for joint frequencies ${ }^{46}$, but also that this kind of phenomenon can be approached by studying the behavior of certain mappings, such as $s \rightarrow F r_{2 ; 5}^{s, s}(3,3)$ which for instance, though continuous, fails to be differentiable at exactly three points in the interval $[1,10]$.

However legitimate it is to suspect that a good deal of hidden information has to be packed in those arrays of numbers in order to account for such 'sensitiveness to the seed' as shown by joint frequencies, it might nevertheless be suggested that the underlying mathematics should be more or less related to the dynamics of the multiplying map from the positive reals onto themselves, $T: x \rightarrow m x$, where $m \in\{2,3, \ldots, 9\}$. This insight not only simplifies the mathematics of the problem by providing a nice synthetic overview of it, but also proves highly beneficial insofar as it allows to focus on the fundamental property lying at the bottom of the above mentioned baffling facts by bringing them back to their ultimate core, the so-called Kronecker-Weyl theorem. 
While looking at every column in the number array as the orbit of the seed under the map $T$, the key idea would then be to bring to the fore the hidden rotations whose underlying workings result in the patterns sifted through computational machinery. This can be done inasmuch as every $T$-orbit of the seed $s$ can only be significantly tracked owing to discrete measurements $\ll T^{n}(s) \gg$ of the iterated values along the orbit, viz. the $T^{n}(s)$, which on the contrary may be supposed to vary continuously with $s$. Insofar as leftmost digits of decimal expansion are insensitive to order shift, since $\ll x \gg=\ll 10 x \gg$, the interval $[1,10)$ is the only one which should be taken heed of, if only rescaled, for the sake of simplicity, so as to fit the unit interval owing to a judiciously chosen function

$$
\begin{aligned}
\psi:[1,10) & \rightarrow[0,1) \\
x & \rightarrow\left(\log _{10} x\right)_{\text {mod. } 1}
\end{aligned}
$$

where the logarithm takes in charge the shrinking while the 'modulo 1' clause guarantees that the values are brought back within the unit interval ${ }^{47}$. The whole procedure can be seen as a means to wind the orbits around the unit circle $\Gamma$ which is obviously isomorphic to the unit interval $[0,1)$ when extremities are pasted together ${ }^{48}$. The $\psi$-function thus provides a topological conjugacy

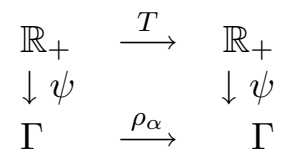

allowing to think of the mapping $T: x \rightarrow m x$ as a rigid rotation $\rho_{\alpha}: x \rightarrow x \oplus \alpha$ on the circle $\Gamma$, where $\bigoplus$ denotes addition modulo 1 and $\alpha=\log _{10}(m)$ (cf. Fig. 10.). The orbit $x$, $T(x), T^{2}(x)$, etc. on the positive real line is thus isomorphic to the orbit $z, \rho_{\alpha} z, \rho_{\alpha}^{2} z$, etc. on the unit circle, and the corresponding sequence of digits

$$
\ll x \gg, \quad \ll T(x) \gg, \quad \ll T^{2}(x) \gg, \quad \cdots
$$

can be thought of as the appropriate tool to study how the points of the orbit are distributed on the circle. The interval $[1,10)$ may indeed be sliced in a series of subintervals

$$
[1,2) \quad[2,3) \quad[3,4) \quad \ldots \quad[9,10)
$$

which can be coiled around the unit circle by being mapped through $\psi$ onto

$$
[0, \log 2) \quad[\log 2, \log 3) \quad[\log 3, \log 4) \quad \ldots \quad[\log 9,1)
$$

so as to provide a criterion to know how the orbit $x, T(x), T^{2}(x)$, etc. is affected when the seed $x$ is varied, since

$$
\ll x \gg=q \quad \text { iff } \quad \psi(x) \in[\log (q), \log (q+1)]
$$

Patterns appear as a result of the discretization of the dynamics of the underlying rotations, for any point in the orbit must by force of combinatorial necessity fall into one interval among the finite number of intervals in which the unit circle is partitioned. At this stage, one can clearly see that since rigid rotations operate at bottom in both cases, Poncelet's theorem and Gelfand's problem are isomorphic. 


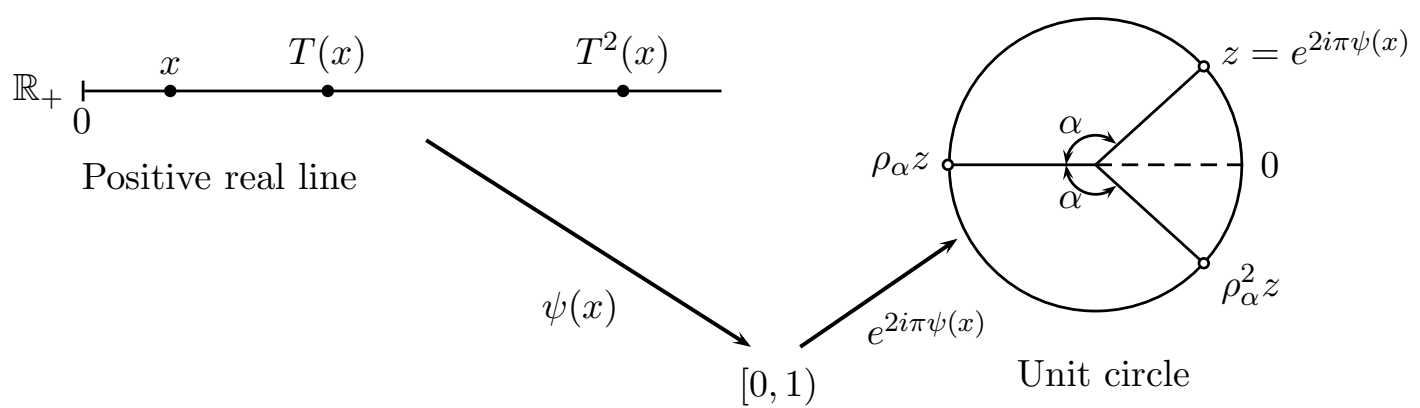

Fig. 10. Winding the positive real line around the unit circle

But in order to account for the frequency phenomena mentioned earlier, something more than Kronecker's theorem is needed. Since every $m$-column in the array of digits is isomorphic to the orbit of a point $z$ on the circle under rotation by $\log (m)$ and since whatever integer $m$ be chosen, the angle of the rotation is an irrational number, the orbit will keep winding around and around the circle indefinitely. But now one might sense that not only such an orbit is dense on the circle, but also that it is uniformly distributed, which, when discretized, means that non only every digit appears infinitely many times in every column, but also that every digit appears with the same frequency in every column independently of the seed. The theorem actually referred to as the 'Kronecker-Weyl theorem' inasmuch as Weyl (1916) significantly extended a proposition previously proved by Kronecker (1884b), is usually stated in the following way ${ }^{49}$ :

Kronecker-Weyl Theorem. Numbers $1, \alpha_{1}, \alpha_{2}, \ldots, \alpha_{n}$ are linearly independent over the rationals if and only if under the action of rotation $\rho_{\alpha_{1}}, \ldots, \rho_{\alpha_{n}}$ on the $n$-dimensional torus, every orbit is uniformly distributed.

But such a compact formulation should not induce the notion of a heavily analytic number theoretic theorem, inescapably relying on the assumption of completed infinities. Kronecker certainely did not understand it this way, at least for the density part which he dealt with. Though the theorem may be formulated in abstract terms involving abstract set theoretic properties such as density, Kronecker nevertheless approached the substance of the theorem in terms of Diophantine approximation.

Kronecker Theorem (1884). If the numbers $1, \alpha_{1}, \alpha_{2}, \ldots, \alpha_{n}$ are linearly independent, $\lambda_{1}, \lambda_{2}, \ldots, \lambda_{n}$ are arbitrary, and $N$ and $\epsilon$ are positive, then there are integers $m>N, p_{1}, p_{2}, \ldots, p_{n}$ such that $\left|m \alpha_{k}-p_{k}-\lambda_{k}\right|<\epsilon$, with $k=1,2, \ldots, n$.

Under this form, the theorem asserts, using Hardy's notation for modulo 1 residue ${ }^{50}$, that there are integers $m$ such that the $(m \alpha)$ 's can be brought simultaneously as near as one would like to the corresponding $\lambda$ 's, however these $\lambda$ 's may be picked out in the unit interval. In the one-dimensional case, Kronecker was even interested in proving more by specifying a bound for the approximation ${ }^{51}$. Now since the fundamental idea is that rational independence of rotation numbers implies the density of the orbits, Kronecker's 
theorem may be rendered "in a more picturesque way" 52 by considering toral rotations. For the sake of simplicity, let us consider only two rotations $\rho_{\alpha}$ and $\rho_{\beta}$ on the unit circle, a toral rotation is thus obtained by conjoining them in the following way

$$
\begin{aligned}
\rho_{\alpha} \times \rho_{\beta}:[0,1) \times[0,1) & \rightarrow[0,1) \times[0,1) \\
\left(z, z^{\prime}\right) & \rightarrow\left(z \oplus \alpha, z^{\prime} \oplus \beta\right)
\end{aligned}
$$

which corresponds geometrically to a mapping on the torus $\Gamma \times \Gamma$ when both rotations are performed simutaneously (cf. Fig. 11). If the orbit under toral rotation $\rho_{\alpha} \times \rho_{\beta}$ of any point in the unit square is represented as the path of a light ray and the sides of the square as reflecting mirrors ${ }^{53}$ (cf. Fig. 12), then Kronecker's theorem asserts that either the path is closed and periodic or it fills out densely the square (or the torus, if one prefers to think of it this way). Whereas optical reflection vividly depicts the 'modulo 1' clause, linear dependence (or independence) of $\alpha$ and $\beta$ corresponds to the rational (or irrational) angle between the intitial direction of the light ray and the side of the square. Besides, with the same picture in one's mind, Kronecker-Weyl theorem would now mean (at least for one sense of the implication) that if $1, \alpha$ and $\beta$ are rationally independent over the rationals, then the sequence of points along the orbit under toral rotation will hit infinitely many times any given finite target $A \times B$ with $A \subset[0,1), B \subset[0,1)$, within the unit square, with a frequency equal to the area of the target since the unit square's area is one (cf. Fig.13).

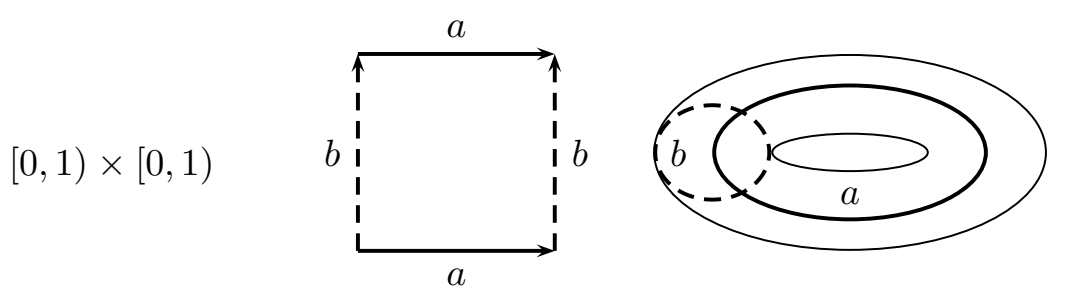

Fig. 11. Toral rotations

At this stage, the rationale for joint frequencies is made perspicuous owing to the fullfledged Kronecker-Weyl theorem. Suffice it to recall that since the unit interval is being sliced into nine subintervals $[0, \log 2),[\log 2, \log 3),[\log 3, \log 4), \ldots[\log 9,1)$, the frequency of any digit $d$ is the same for every column independently of the seed, simply because it is equal to the length of the corresponding interval $[\log d, \log (d+1))$, namely $\log \left(\frac{d+1}{d}\right)$, which does depend neither on the multiplier nor on the seed. 

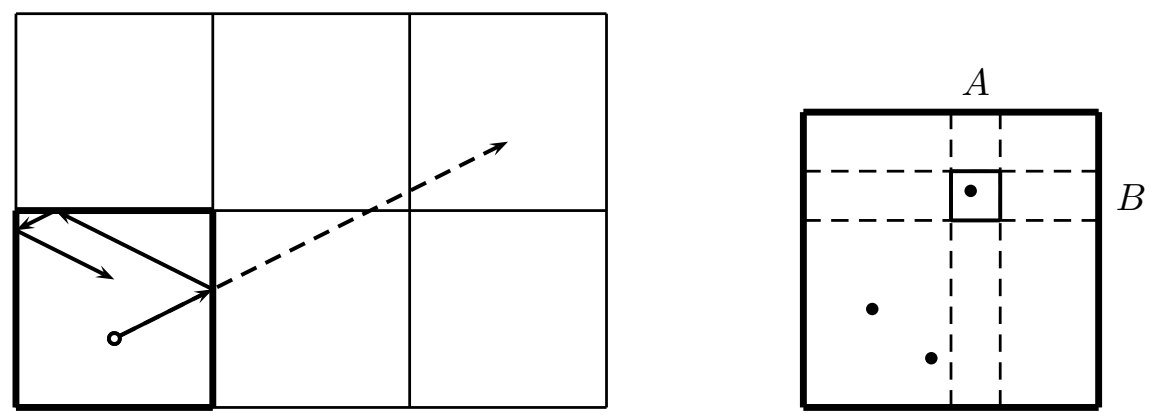

Fig. 12. The 'Kronecker ' part of K-W theorem Fig. 13. The 'Weyl' part of K-W theorem

But even more strikingly, one eventually grasps the reason why some of the columns of the array of digits are independent and why some others are not. Since for instance, as King (1994) explains, $1, \log 2$ and $\log 3$ are rationally independent, the joint frequency of having in a one row a digit $d$ in column 2 and a digit $d^{\prime}$ in column 3 is equal to the area of the target square which is the product of both frequencies considered separately ${ }^{54}$, and the nature of the seed does not even come into the picture. Reversely, the ultimate gist of the colums 4 and 5 being utterly non independent, as revealed by heuristic exploration, boils down to a simple arithmetical truth, namely $5^{2} .4=100$, or equivalently, though much more perspicuously assuming Kronecker-Weyl theorem, $2 \log 5+\log 4=\log 100=2$.

\section{Final remarks on computational understanding}

Is this search for arithmetical facts which, though enshrined in analytic contexts, nevertheless provide the utlimate rationale for them, faithful to what Kronecker had in mind when proving his part of the theorem? Without pretending to give an overview, even if concise, of the history of the Kronecker-Weyl theorem, a few hints might be helpful to suggest that this question should be answered positively. Kronecker obtained his theorem as the outcome of a method which he had developed so as to handle questions relating to systems of periods of functions. Two memoirs, (Kronecker, 1884a) and (Kronecker, 1884b), were published in rapid succession, which do overtly link function-theoretic concerns with Diophantine approximation problems. As is often the case, Kronecker's most productive thoughts stem here from Jacobi's legacy, and more specifically from the way Jacobi (1834) dealt with the question of how many independent periods a function may have, which were first paid heed of in elliptic function theory. On the base of arithmetical facts, Jacobi had actually proved that if a function has two distinct periods which cannot be brought back to only one, then the ratio of these two is no more rational than it is irrational, so that it has to be a complex quantity. But moreover and mainly, he had devised "a precise algorithm" 55 owing to which, assuming that a function may have three independent complex periods, one could build an indefinitely long series of further periods whose imaginary as well as real parts could be 
made less than any arbitrary quantity, though they do not utterly vanish. Which, being impossible, yields the conclusion that there is no such thing as a triply periodic function. In his own memoir, Kronecker refers from the outset at Jacobi's in the following terms: "Jacobi concludes his developments on the number of periods a function of a complex variable might have by these words : 'Therefrom [viz. from the algorithm mentioned above] it has been demonstrated in all cases that if the proposed function enjoys three periods, either they are composed of only two of them, or the function has a period which is less than any given quantity. Which is absurd so there is no triply periodic function'56. Jacobi stops here and omits to give more in detail the reason for the alleged absurdity of the consequences that can be drawn from the assumption of triple periodicity" 57 . Kronecker's theorem aims at nothing else than extracting this very reason as a fundamental arithmetical fact.

It has thus been argued that a persuasive case can be made that Jacobi's model of Poncelet's theorem is illustrative of two major features of Kroneckerian finitism. On the one hand, the interpretation of the closure conditions for Poncelet's polygons in terms of integral multiplication of elliptic functions makes clear that finite patterns do emerge through algorithmic fine-tuning, inasmuch as the whole computational scheme is mirrored in a geometrical setting. Halphen's construction shows indeed in a perspicuous way that the closure holds only for singular positions of the inner circle which, when shifted, even if slightly, from these distinguished spots, do throw the Poncelet traverse off balance by turning it into a never-ending ongoing polygon indefinitely winding around the circle. But on the other hand, visual evidence is also thereby adduced for the belief, firmly held by Kronecker, that completed infinities can utterly be dispensed with in mathematics, since it provides one with a vivid sense of this dispensability by making clear that the Poncelet's polygon may keep filling out densely the circle with vertices, owing to the 'arithmetical essence' of what an irrational rotation boils down to. Therefore as Kummer once put it in a letter to H. A. Schwartz, while refering to a conviction which he and Kronecker had in common, "the effort to create enough individual points to fill out a continuum - that is, enough real numbers to fill out a line [or the unit circle] - is as vain as the ancient efforts to prove Euclid's parallel postulate" 58 .

In conclusion, Jacobi's model foreshadows how Kroneckerian finitism is to be thought of as grounded in an acute sense of a 'deeper-than-meets-the-eye' arithmetization of mathematics, especially regarding how transcendent elements should be dealt with. Nevertheless, in order to conceptually characterize in a more general way the notion of discreteness of the intended patterns that was appealed to in this paper, and the corresponding algorithmic fine-tuning procedures thus required, one should follow the second path invoked above, namely generalize Jacobi's model by shifting to complex multiplication and thus grasp Kronecker's methodological tenet in its proper setting by making clear how his brand of finitism is rooted in his quest for singular moduli of elliptic functions. For the sake of clarity, these issues will be specifically addressed in a separate paper ${ }^{59}$, whereas hopefully this one should be seen as providing only a vivid, though restricted, instantiation thereof. Eventually, it may be suggested that the widely held divide between conceptual and algorithmic mathematics might be challenged to a certain extent since Jacobi's model fleshes out how 
insightful understanding might be gained through computations, instead of being marred by them, inasmuch as they allow us to spot mathematically significant patterns.

\section{Acknowledgements}

I thank Leon Horsten, Wilfried Sieg and Jamie Tappenden for comments on a first draft of this paper, Mic Detlefsen and Sean Walsh for stimulating exchanges about some of the issues dealt with here.

\section{Notes}

${ }^{1}$ Cf. (Reed, 1995, p. 98) who takes heed of this vexing question.

${ }^{2}$ As fleshed out for instance in (Kronecker, 1887).

${ }^{3}$ (Kronecker, 1901, 2) : "Andrerseits würde die durch die Gaußische Definition gefordete Abgrenzung der Arithmetik gegen die Analysis die kontinuirlichen Grössen und die Anwendung der auf sie gegründeten Methoden in der Hauptsache dem Bereiche der Zahlentheorie entziehen. Eine derartige Beschränkung erschien allerdings geboten zu einer Zeit, als man solche Quantitäten noch mehr geometrisch faßte; sie ist aber hinfällig geworden, seitdem man neuerdings sich bemüht, viele der Geometrie oder Mechanik entstammende Größen ohne Rücksicht auf diese Entstehung zu definieren, womit dan sofort ihr rein arithmetisches Wesen in den Vordergrund tritt. Definirt man z. B. die aus der Geometrie herrührende Transcendente $\pi$ etwa durch die Leibniz'sche Reihe

$$
\frac{\pi}{4}=1-\frac{1}{3}+\frac{1}{5}-\frac{1}{7}+\ldots=\sum_{n=0}^{\infty} \frac{(-1)^{n}}{2 n+1}
$$

so ergiebt sich aus dieser grade eine der schönsten arithmetischen Eigenschaften der ungeraden Zahlen, nämlich eben die, jene geometrische Irrationalzahl zu bestimmen; in diesem Sinne ist wohl jenes bekannte Wort : 'numero impari deus gaudet' zu verstehen. Die Glieder dieser Reihe sind nämlich, um das etwas näher auszuführen, arithmetisch wohl unterschieden : sie haben das positive oder negative Vorzeichen, je nachdem ihre Nenner durch 4 geteilt den Rest 1 oder 3 lassen. Wir haben hier also eine Definition der Transcendenten $\pi$ von durchaus zahlentheoretischem Charakter."

${ }^{4}$ Cf. (Detlefsen, 2008, p. 254) who comments on this excerpt from Gauss's Anzeige, Theoria residuorum biquadraticorum (1831).

${ }^{5}$ Cf. (Smadja, forthcoming (a)) for a historical assessment of the significance of Kronecker's emphasis on singular moduli for arithmetization in the case of the Lemniscate. Following some hints given by $\mathrm{C}$. L. Siegel, Kronecker's conception is outlined by tracing back complex multiplication to the prehistory of elliptic function theory, where the pattern can be grasped 'in nuce'.

${ }^{6}$ Cf. Kronecker's letter to Dirichlet dated May, 17th, 1857, (Kronecker, 1895-1931, Vol. V, p. 419) : "The two main episodes of my work dealt with the complex multiplication of elliptic functions and the theory of the most general ideal numbers. As regards the first theme, I have already communicated my results in december. In the first three months of this year, I have found even more similar results, I have proved everything rigorously and really concluded completely the whole investigation. I found the little mathematical field which I have explored throughout very interesting in many respects. The elegance of the results and the simplicity of the methods of proof, the connection of analysis and number theory, the wider prospects which these things allow, all this united to spur and then reward my zeal for this investigation.". "Die beiden Hauptepisoden meiner Arbeiten betrafen die complexe Multiplication der elliptischen Functionen und die Theorie der allgemeinsten idealen Zahlen. In Bezug auf das erste Thema habe ich Ihnen meine 
ersten Resultate schon im December hier mitgetheilt. Ich habe in den ersten drei Monaten dieses Jahres noch viel ähnliche Resultate gefunden, Alles streng bewiesen und überhaupt die betreffende Untersuchung eigentlich abgesclossen. Das dabei durchforschte kleine mathematische Gebiet war mir in vieler Beziehung äußert interessant. Die Eleganz der Resultate und die Einfachheit der Beweismethoden, der Zusammenhang der Analysis und Zahlentheorie, die weiteren Aussichten, die die Sachen gewähren - alles das vereinigte sich, um meinen Eifer in der Untersuchung anzuspornen und nachher zu belohnen".

${ }^{7}$ Cf. (Smadja, forthcoming (c)) for a more general discussion of Kronecker's conception of arithmetization in the light of his work on complex multiplication of elliptic functions.

${ }^{8}$ (Thurston, 1994, p. 3).

${ }^{9}$ (Thurston, 1994, p. 2).

${ }^{10}$ (Thurston, 1994, p. 5).

${ }^{11}$ Cf. (Poncelet, 1822, Section 566).

${ }^{12}$ (Jacobi, 1828, p. 285) : "In dieser Form der Gleichungen springt es sogleich in die Augen, dass sie mit denjenigen übereinkommen, welche zur Vervielfachung der elliptischen Transcendenten aufgestellt werden."

${ }^{13}$ (Halphen, 1886-1891, vol. I, chap. I, p. 23.).

${ }^{14} \mathrm{Cf}$. (Smadja, forthcoming (b)) for a precise sketch of these computations.

${ }^{15}$ Elliptic integrals in Jacobi's notations, namely $\int_{0}^{x} \frac{d x}{\sqrt{1-x^{2}} \sqrt{1-k^{2} x^{2}}}$, are obtained from those in Legendre's normal form, $\int_{0}^{\phi} \frac{d \phi}{\sqrt{1-k^{2} \sin ^{2} \phi}}$, by the substitution $x=\sin \phi$, where $\phi=a m u$ is the so-called amplitude. Jacobi then defines an elliptic function as a 'sinus amplitudinis' by inverting the corresponding integral $u=\int_{0}^{x} \frac{d x}{\sqrt{1-x^{2}} \sqrt{1-k^{2} x^{2}}}$ into $x=\sin a m u$.

${ }^{16}$ (Bos et al., 1987, p. 359).

${ }^{17}$ Cf. (Poncelet, 1862, p. 482-483) : "Quel que soit le mérite d'un pareil rapprochement et quoiqu'on sache par les plus anciens travaux d'Euler, de Legendre, de Jacobi et d'Abel que les intégrales nommées elliptiques ont entre elles des relations purement algébriques ou géométriques, il n'en paraît pas moins peu naturel et encore moins philosophique, de tirer directement de la connaissance de ces travaux, (...) la démonstration, a posteriori, de théorèmes généraux aussi simples de la géométrie".

${ }^{18}$ Cf. (Poncelet, 1862, p. 482) : "les fonctions elliptiques n'ayant qu'une valeur de forme purement implicite ou algorithmique, qui fait dépendre, contrairement à l'ordre logique et rationnel, le simple du composé ou du transcendant".

${ }^{19}$ (Bos et al., 1987, p. 322).

${ }^{20}$ Cf. (Jacobi, 1828, p. 293).

${ }^{21}$ (Griffiths, 1978, p. 32).

${ }^{22}$ More precisely, Cayley brings the quadratic equations of the conics, viz. $U=0$ and $V=0$, to normal form, by mapping them respectively onto $a x^{2}+b y^{2}+c z^{2}=0$ and $x^{2}+y^{2}+z^{2}=0$, then forms the pencil $U+\zeta V=0$, viz. $(a+\zeta) x^{2}+(b+\zeta) y^{2}(c+\zeta) z^{2}=0$, and makes the discriminant equal to 0 , viz. $\left|\begin{array}{ccc}a+\zeta & 0 & 0 \\ 0 & b+\zeta & 0 \\ 0 & 0 & c+\zeta\end{array}\right|=(a+\zeta)(b+\zeta)(c+\zeta)=0$.

${ }^{23}$ Cf. (Loria, 1889, Note (3), p. 20-22).

${ }^{24} \mathrm{Cf}$. (Griffiths, 1978, p. 40) : "... we consider the pencil of conics $D_{t}=\{t C(x)+D(x)=0\}$ [with $x=\left[\lambda_{0}, \lambda_{1}, \lambda_{2}\right]$ in homogeneous coordinates] passing through the four base points $x_{i}$ [namely the four points of intersection of $C$ and $D]$. The determinant $\operatorname{det}(t C(x)+D(x))$ is a cubic polynomial in $t$ with non-zero roots $t_{i}(i=1,2,3)$. For $t \neq t_{i}$ we draw the tangent line to $D_{t}$ through $x_{0}$ meeting $C$ in a unique residual point $x(t)$. It is easy to see that $t=t_{i}$ is mapped into $x_{i}$ (with suitable indexing), and since $D_{\infty}=C$ the value $t=\infty$ is mapped to $x_{0}$. Taking $t=0$ we see that $t=0$ corresponds to $x$, so that in summary : The elliptic curve $E$ is birationally equivalent to the Riemann surface of the algebraic function $\sqrt{\operatorname{det}(t C(x)+D(x))}$ with the origin $\mathcal{O}$ corresponding to $t=\infty$ and the point $p=(\underline{x}, \underline{\xi})$ to one of the two points lying over $t=0 . "$ 
${ }^{25}$ Cf. (Halphen, 1886-1891, Vol. I, chap. X, p. 368). Given a unicursal plane curve $C$ so that every value of the parameter $x$ corresponds to one and only one point on the curve, every quadratic equation in two variables $x$ and $x^{\prime}$ can be seen as a relation between the two corresponding points on the curve. Suppose now that the equation is quadratic in $x^{\prime}$, for every point $x$ there are two corresponding points $x^{\prime}$, choose one of them as $x_{1}$. If the quadratic equation is symmetric, one can proceed the reverse way, the point $x_{1}$ has two corresponding points, the previous $x$ plus another one. Call it $x_{2}$. And so on and so forth. By iteration, one obtains a variable polygonal line which is completely determined by its starting point $x$ and is inscribed in the unicursal curve $C$. So any symmetric doubly quadratic equation expresses the relation between the extremities of a variable chord inscribed in a first conic and tangent to a second one.

${ }^{26}$ Cf. (Halphen, 1886-1891, Vol. I, chap. IX, p. 334).

${ }^{27}$ Cf. (Walsh, forthcoming) for a comment of Kronecker's 1891 lecture on the number concept in mathematics, in the light of a precise discussion of the arithmetization of the concept of volume as an instance of analytic invariant.

${ }^{28}$ (Kronecker, 1891, p. 23-24).

${ }^{29}$ (Kronecker, 1891, p. 25-26) : "Ich unterscheide die Invarianten als arithmetische, algebraische und analytische Invarianten nach der Methode, durch welche sie aus den Elementen eines Systems hergeleitet werden". Cf. (Walsh, forthcoming) for a perusal of Kronecker's own explications (1891) of such notions of equivalence, class and invariants as applied to the first steps of the theory of quadratic forms.

${ }^{30} \mathrm{Cf}$. (Walsh, forthcoming) for an assessment of this philosophical point.

${ }^{31}$ (Loria, 1889, p. 22) : "una semplicissima considerazione palesa subito la ragione di questo fatto algebrico".

${ }^{32}$ Cf. (Kronecker, 1887, p. 249) for the quote from Jacobi's letter to Alexander von Humboldt where, after an ancient Greek, mathematicians are compared to the Lotus-eaters in Homer's Odyssey, insofar as the passion for mathematical knowledge is as furious as the addiction to the fruit of the lotus.

${ }^{33}$ Cf. (Hermite, 1905-1917, Vol. IV, p. 340-341) : 'L'œuvre capitale de M. Kronecker est d'avoir trouvé, dans la théorie de la transformation complexe, une source d'accès plus difficile, mais infiniment plus féconde pour l'Arithmétique. (...) M. Kronecker établit qu'à chaque classe de forme quadratique correspond un module singulier qui permet la multiplication complexe (...) M. Kronecker a mis en complète évidence que la théorie des formes quadratiques, de déterminant négatif, a été une anticipation de la théorie des fonctions elliptiques".

${ }^{34}$ Cf. (Smadja, forthcoming (a)) and (Smadja, forthcoming (c)).

${ }^{35} \mathrm{Cf}$. in particular (Smadja, forthcoming (c)) for a discussion of these issues.

${ }^{36}$ More precisely, since elliptic functions are doubly periodic, one can associate a parallelogram of periods to any elliptic integral, so that the transformation of such elliptic integrals is governed by the arithmetic operating on the corresponding lattices in the complex plane.

${ }^{37}$ See (Tabachnikov, 2005) for a complete survey of the whole field.

${ }^{38}$ Assuming a transformation $R$ mapping a measure space $(X, \mu)$ onto itself, the measure $\mu$ is said to be invariant if for every subset $A \subset X$, the property $\mu(R(A))=\mu(A)$ holds. In the particular case in which $R$ is taken to be the rigid rotation on the circle, denoted $\rho_{\alpha}$, with $\alpha \in \mathbb{R}$, and the measure is the usual arclength $\lambda$ on the circle, the measure is obviously $\rho_{\alpha}$-invariant.

${ }^{39}$ Cf. (King, 1994, p. 614).

${ }^{40}$ The proof may be outlined as follows. Let $\rho_{\alpha}$ be an irrational rotation corresponding to $\alpha \in \mathbb{R}$. For every $n$, the unit circle $\Gamma=[0,1)$ may be partitioned into $n$ subintervals $\left[0, \frac{1}{n}\right),\left[\frac{1}{n}, \frac{2}{n}\right), \ldots,\left[\frac{n-1}{n}, 1\right)$. Considering the collection of $n+1$ points $0, \rho_{\alpha}(0), \rho_{\alpha}^{2}(0), \ldots, \rho_{\alpha}^{n}(0)$, by the 'pigeonhole' principle, two of these, say for instance $\rho_{\alpha}^{k}(0)$ and $\rho_{\alpha}^{l}(0)$, with $0<k<l<n$, fall into the same subinterval $\left[\frac{p}{n}, \frac{p+1}{n}\right)$ with $0<p<n-1$. Now the iterated rotation $\rho_{\alpha}^{m}$, with $m=l-k$, obviously maps $\rho_{\alpha}^{k}$ to $\rho_{\alpha}^{l}$, and, since it is a rigid rotation, both 0 and $\rho_{\alpha}^{m}(0)$ belong to $\left[0, \frac{1}{n}\right)$. Therefore since the length of the subintervals can be diminished at will by choosing $n$ as large as one wishes, the orbit of 0 under the rotation is dense in the unit circle.

${ }^{41}$ (Rota, 1986, p. 61). 
${ }^{42}$ (Rota, 1986, p. 62).

${ }^{43}$ Ibid.

${ }^{44}$ (Kronecker, 1901, p. 2).

${ }^{45}$ (King, 1994, p. 625) refers the problem of the "frequencies of $2^{n "}$ to (Avez, 1966, p. 37) where it is attributed to Gelfand.

${ }^{46}$ Joint frequencies might be denoted by enriching the indices in order to account for the seed, so that $\mathrm{Fr}_{3: 5 ; 7}^{s, s^{\prime}, s^{\prime \prime}}(2,1,7)$ for instance denote the frequency of rows having a ' 2 ' in column 3 , a ' 1 ' in column 5 and a ' 7 ' in column 9, when the seeds $s, s^{\prime}$ and $s^{\prime \prime}$ are provided respectively in the columns 3,5 and 7 .

${ }^{47}$ Since $\log _{10} 10^{p \cdot x}=p+\log _{10} x$, one has to take the values modulo 1 in order to get rid of $p$.

${ }^{48} \mathrm{As}$ is well known, $\psi:[0,1) \rightarrow \Gamma, \theta \rightarrow e^{2 i \pi \theta}$ does the job.

${ }^{49}$ Cf. (King, 1994, p. 626) for the connections of the Kronecker-Weyl theorem with ergodic theory.

${ }^{50} \mathrm{Cf}$. (Hardy et al., 1998, p. 156), where $[x]$ denotes the integral part of $x$, viz. the greatest integer less than $x$, and hence $(x)=x-[x]$ the residue of $x$ modulo 1 .

${ }^{51}$ Cf. (Hardy et al., 1998, p. 377) for a faithful presentation, namely in terms of Diophantine approximation, of Kronecker's theorem, which in one dimension states that : "if $\alpha$ is irrational, $\lambda$ arbitrary, and $N$ positive, then there is an $n>N$ and a $p$ for which $|n \alpha-p-\lambda|<\frac{3}{n}$ ".

${ }^{52}$ Ibid.

${ }^{53}$ Cf. (Hardy et al., 1998, p. 379) where Kronecker's theorem is applied to the 'mirror problem' attributed to König and Szücs.

${ }^{54}$ In this case, the area of the target square $[\log d, \log (d+1)) \times\left[\log d^{\prime}, \log \left(d^{\prime}+1\right)\right)$, and hence the joint frequency, is equal to $\log \left(\frac{d+1}{d}\right) \cdot \log \left(\frac{d^{\prime}+1}{d^{\prime}}\right)$.

${ }^{55}$ (Jacobi, 1834, p. 31).

${ }^{56}$ This quote is taken from (Jacobi, 1834, p. 32).

${ }^{57}$ (Kronecker, 1884b, p. 49-50, footnote $\left.\left({ }^{* *}\right)\right)$ : "Jacobi schliesst im $\S 4$ seine Entwickelungen über die mögliche Anzahl der Perioden von Functionen einer complexen Variablen mit den Worten : 'Unde omnibus casibus evictum est, si functio proposita tribus periodis gaudeat, aut eas e duabus componi, aut eam habere indicem omni data quantitate minorem. Quod cum absurdum sit, functio tripliciter periodica non datur.' Jacobi bleibt hierbei stehen, und unterlässt es, die behauptete Absurdität durch die aus der Voraussetzung dreifacher Periodicität zu ziehenden erschöpfenden Folgerungen näher zu begründen."

${ }^{58}$ Cf. (Edwards, 2009, p. 14.) where this excerpt from a recently discovered letter by Kummer to H. A. Schwartz, dated 15 March 1872, is quoted. The addition and emphasis between brackets are mine.

${ }^{59}$ Cf. (Smadja, forthcoming (c)).

\section{References}

Avez, A., (1966), Ergodic Theory of Dynamical Systems, Vol. 1., Minneapolis, University of Minnesota.

Bos, H.J.M., Kers, C., Oort, F. and Raven, D.W., (1987), Poncelet's closure theorem, Expositiones Mathematice 5 , pp. 289-364.

Cayley, A. (1853a), Note on the Geometrical Representation of the Integral $\int \frac{d x}{\sqrt{(x+a)(x+b)(x+c)}}$, Philosophical Magazine V, 4th series, pp. 281.

Cayley, A. (1853b), Note on the Porism of the In-and-Circumscribed Polygon, Philosophical Magazine VI, 4th series, pp. 99.

Cayley, A. (1861), On the Porism of the In-and-Circumscribed Polygon, Philosophical Transactions of the Royal Society of London V, 151, I Part, pp. 225.

Detlefsen, M., Purity as an Ideal of Proof, in The Philosophy of Mathematical Practice, ed. Paolo Mancosu, Oxford University Press. pp. 248-277.

Edwards, H. M., (1974), Riemann's Zeta Function, New-York, Academic Press. 
Edwards, H. M., (1977), Fermat's Last Theorem. An Genetic Introduction to Algebraic Number Theory, Springer.

Edwards, H. M., (1980), The Genesis of Ideal Theory, Arch. Hist. Ex. Sci. 23, pp. 321-378.

Edwards, H. M., (1983), Dedekind's Invention of Ideals, Bull. Lond. Math. Soc. 15, pp. 8-17, Reprinted in MAA Studies in Mathematics, Volume 26, Studies in the History of Mathematics, Esther R. Phillips, ed., MAA, 1987.

Edwards, H. M., (1987), An Appreciation of Kronecker, Mathematical Intelligencer 9, 1, pp. 28-35.

Edwards, H. M., (1988), Kronecker's Place in History, in: History and Philosophy of Modern Mathematics, W. Aspray and P. Kitcher, eds., Minnesota Studies in the Philosophy of Science, vol. 11, Univ. of Minn. Press.

Edwards, H. M., (1989), Kronecker's Views on the Foundations of Mathematics, in: The History of Modern Mathematics, D. E. Rowe and J. McCleary, eds., vol. 1, 67-78, Academic Press.

Edwards, H. M., (1995), Kronecker on the Foundations of Mathematics, in: From Dedekind to Gödel, Jaakko Hintikka, ed., Kluwer, 1995.

Edwards, H. M., (2005), Essays in Constructive Mathematics, New-York-Berlin, Springer

Edwards, H. M., (2007), Kronecker's Fundamental Theorem of General Arithmetic, in: Episodes in the History of Modern Algebra, J. Gray, K. H. Parshall, eds., American Mathematical Society.

Edwards, H. M., (2008), The Algorithmic Side of Riemann's Mathematics, Communication at the Joint AMS-MAA Meetings, San Diego.

Edwards, H. M., (2009), Kronecker's Algorithmic Mathematics, Mathematical Intelligencer 31, 2, pp. 8-17.

Gauss, C. F., (1863-1933), Werke, 12 vols, Leipzig-Berlin.

Griffiths, P. A., (1976), Variations on a theorem of Abel, Inventiones Math. Vol. 25, pp. 321-390.

Griffiths, P. A., Harris, J., (1978), OnCayley's explicit solution to Poncelet's Porism, L'Enseignement Mathmatique. Vol. 24, pp. 31-40.

Halphen, G.-H., (1886-1891), Traité des fonctions elliptiques et de leurs applications, Gauthier-Villars, Paris.

Hardy, G. H. and Wright, E. M., (1998), An Introduction to the Theory of Numbers, Oxford, Clarendon Press, 5th edition.

Hermite, C., (1905-1917), Euvres, Gauthier-Villars, Paris. Edited by Emile Picard.

Jacobi, C.G.J., (1828), Ueber die Anwendung der elliptischen Transcendenten auf ein bekanntes Problem der Elementargeometrie, Journal für die reine und angewandte Mathematik Vol. 3, pp. 376-389, reprinted in Jacobi, C.G.J, Gesammelte Werke, I, p. 277-293.

Jacobi, C.G.J., (1834), De functionibus duarum variabilium quadrupliciter periodicis, quibus theoria transcendentium abelianarum innititur, in Jacobi, C.G.J, Gesammelte Werke, II, p. 23-50.

King, J. L., (1994), Three Problems in Search of a Measure, American Math. Monthly Vol. 101, pp. 609-628.

Kronecker, L., (1857), Ueber die elliptischen Functionen, für welche complexe Multiplication stattfindet, Monatsberichte der Königlich Preussischen Akademie der Wissenschaften zu Berlin pp. 363-372, reprinted in Werke, IV, pp. 185-195.

Kronecker, L., (1884a), Die Periodensysteme von Functionen reeller Variablen, Monatsberichte der Königlich Preussischen Akademie der Wissenschaften zu Berlin pp. 1071-1080, reprinted in Werke, III (i), pp. 31-46.

Kronecker, L., (1884b), Näherungsweise ganzzahlige Auflösung linearer Gleichungen, Monatsberichte der Königlich Preussischen Akademie der Wissenschaften zu Berlin pp. 1179-1193,1271-1299, reprinted in Werke, III (i), pp. 47-110.

Kronecker, L., (1887), Ueber den Zahlbegriff, Journal für die reine und angewandte Mathematik Vol. 101, pp. 337-355, reprinted in Werke, III, pp. 249-272.

Kronecker, L., (1891), Ueber den Begriff den Zahl in der Mathematik, Revue d'Histoire des Mathmatiques 7, (2), (2001) pp. 219-275. The unpublished manuscript of the 1891 lecture has been transcribed and edited by Jacqueline Boniface and Norbert Schappacher.

Kronecker, L., (1895-1931), Werke, Leipzig, K. Hensel (ed), 5 vols. 
Kronecker, L., (1901), Vorlesungen über Zahlentheorie, Teubner, Leipzig, K. Hensel (ed).

Loria, G. (1889), I poligoni di Poncelet, Torino, 1889.

Poncelet, J.-V., (1822), Traité des propriétés projectives des figures, Paris.

Poncelet, J.-V., (1862), Applications d'analyse et de géométrie, qui ont servi, en 1822, de principal fondement au Traité des propriétés projectives des figures, Paris.

Reed, D., (1995), Figures of Thought. Mathematics and Mathematical Texts, Routledge, London and NewYork.

Pasch, M, Rosanes, J., (1869), Journal für die reine und angewandte Mathematik Vol. 70, pp. 169-174.

Kac, M., Rota, G.-C. and Schwartz, J. T., (1986), Discrete Thoughts, Essays on Mathematics, Science and Philosophy, Boston, Birkhäuser.

Smadja, I., (forthcoming (a)), La lemniscate de Fagnano et la multiplication complexe. (submitted to the Revue d'Histoire des Mathématiques, Paris, SMF.)

Smadja, I., (forthcoming (b)), Halphen lecteur de Jacobi. Les polygones de Poncelet la lumière des fonctions elliptiques, in Histoire des courbes, ed. Ph. Abgrall, Paris, Oriens-Occidens.

Smadja, I., (forthcoming (c)), The Discrete and the Singular. Kronecker on Arithmetization. (manuscript)

Tabachnikov, S., (2005), Geometry and Billiards, AMS, 2005.

Thurston, W. P., (1994) On proof and progress in mathematics Bulletin of the American Mathematical Society Vol. 30, 2, April, pp. 161-177.

Walsh, S., (forthcoming), Kronecker and the Arithmetization of the Concept of Area. (manuscript)

Weyl, H. (1916), Ueber die Gleichverteilung von Zahlen mod. Eins, Mathematische Annalen Vol. 77, pp. 313-352. 
VisualKronecker3.tex; 1/08/2009; 18:21; p.26 